\title{
Potencialidades en comunicación científica y elementos de virtud de investigadores mexicanos
}

Potentialities in scientific communication and elements of virtue in Mexican researchers

\author{
Yesenia Mendoza-Villalobos, Javier TARAngo, José Refugio Romo-GonzÁlez \\ Universidad Autónoma de Chihuahua (México), Avenida Universidad s/n, Ciudad Universitaria, 31174, Chihuahua, México. \\ yesymv@hotmail.com, jtarango@uach.mx, jromo@uach.mx
}

\begin{abstract}
Resumen
Este artículo plantea como objetivo, identificar la influencia de la virtud académica en el desarrollo de potencialidad científica (PC) en la comunicación científica (CC) por investigadores mexicanos. Para ello, el modelo toma como referencia los elementos de la virtud aristotélica y los traspola a la capacidad de CC, bajo tres elementos de evaluación: (1) el carácter (conocimiento y razón); (2) la voluntad (intencionalidad); y (3) el hecho moral (acciones realizadas). Para la recolección de los datos, se aplicó un cuestionario a una muestra aleatoria de 183 investigadores provenientes de universidades públicas estatales mexicanas, en relación a sus condiciones de actividad científica y su acercamiento a los elementos de la virtud. El análisis de datos (con un nivel de confianza del 95\%), encontró una alta significancia del estadístico de prueba, donde se explica que, a mayor presencia de elementos de la virtud académica de cada investigador, lo potencian a comunicar ciencia de forma amplia en cantidad y de manera sistemática.
\end{abstract}

Palabras clave: Investigación científica. Producción científica. Comunicación científica. Potenciamiento en investigación científica. Virtud académica. México.

\section{Introducción}

Cuando se le pide a un investigador científico que defina su función y las razones del porqué llegó a desempeñarse en esta actividad, existen respuestas poco precisas. Las actividades profesionales de un investigador científico tienen dos vertientes (1) actividad desarrollada con fines laborales, con propósitos de obtener ingresos y solventar su vida personal y familiar; y (2) búsqueda del conocimiento, la curiosidad intelectual y la resolución de problemas a través de diversos modelos de experimentación. Ambas actividades resultan complementarias e imposibles de separar.

Armas Faris (2011) y Tomić et al. (2021), consideran que la investigación es una actividad personal, modesta o brillante, que exige cualidades, tales como la minuciosidad, fijeza, paciencia y voluntad, sopesando, además, la integridad ética en la investigación. Los autores analizan las virtudes epistémicas en el conocimiento científico

\begin{abstract}
This article sets out as a goal, to identify the influence of academic virtue in the development of scientific potentiality (PC) in scientific communication (CC) by Mexican researchers. To do this, the model takes the elements of Aristotelian virtue as a reference and transposes them to the capacity for scientific communication, under three elements of evaluation: (1) character (knowledge and reason); (2) the will (intentionality); and (3) the moral fact (actions taken). For data collection, a questionnaire was applied to a random sample of 183 researchers from Mexican state public universities, in relation to their conditions of scientific activity and their approach to the elements of virtue. The data analysis (with a confidence level of $95 \%$ ), found a high significance of the test statistic, where it is explained that, the greater the presence of elements of the academic virtue of each researcher, they empower him to communicate science in a broad way in quantity and systematically.
\end{abstract}

Keywords: Scientific research. Scientific production. Scientific communication. Potential in scientific research. Academic virtue. Mexico.

basadas en la atención, la humildad intelectual, el rigor y orden intelectual. Los elementos antes mencionados definen de forma más precisa el concepto de virtud en la investigación y comunicación científica (CC), procesos que se desarrollan a lo largo de la vida y que se convierte en crecimiento, buscando con ello la adquisición de un equilibro entre querer, saber y poder hacer ciencia de forma sistemática, con lo cual se obtiene un desarrollo, como en cualquier otra actividad profesional, hasta convertirse en acciones cotidianas.

La virtud se basa en actos observables, por tanto, no debe ser una acción teórica sino práctica (Berg, 2020; Näsman y Nyholm, 2020). Para ello, se vuelve necesario describir la relación entre las acciones y las virtudes, por lo que se considera al investigador científico como necesariamente una persona virtuosa, lo que implica ejercicio de las facultades, desarrollo de actividades 
científicas basadas en lo apropiado y justo, además de una adecuada disposición interior (Garcés Giraldo y Giraldo Zuluaga, 2013).

Al conjuntar a la CC con la virtud, el investigador científico juega un papel fundamental en las universidades y los convierte en los actores principales. En el caso de las universidades en Latinoamérica, el número de investigadores científicos reconocidos como tales son insuficientes, donde su legitimación está basada en la medición de la cantidad y calidad de productos científicos que generan y que son comunicados en medios de publicaciones de reconocido prestigio científico, entendido esto, a través de artículos científicos, libros, capítulos de libro, direcciones de tesis, patentes y normas, por mencionar los principales.

El objetivo planteado en esta investigación es determinar si los investigadores científicos mexicanos reflejan mayor virtud en la CC, dependiendo de su nivel de reconocimiento, mostrando mayor acercamiento a los tres elementos de la virtud: presencia suficiente en el saber (conocimiento y razón), querer (intencionalidad) y hacer (acciones realizadas).

\section{Virtud, educación y ciencia: transdisciplinariedad en la generación de conocimiento}

Conjugar los términos de virtud, educación y ciencia representa identificar elementos de cohesión y sinergia, considerando con ello el planteamiento de Platón, quien consideró que el objetivo de la educación es la virtud y el deseo de convertirse en un buen ciudadano. La sinergia de estos términos, para Vélez-Ramírez (2008), radica en que el fin primario de la universidad debe ser el incremento del bien común virtuoso, por ello se debe aumentar la virtud personal.

La educación es sopesada como la pieza clave para formar el carácter y trabajar sobre la virtud como tema emergente (Burlando, 2012; Fowers et al., 2020), pero además es considerada como una manera de formar hábitos, lo cual, conlleva al conocimiento y su generación (Barrantes Montero, 2013). Por tanto, un investigador científico, requiere obtener un carácter masivo y de equidad, combinación de estudio y trabajo, participación democrática y abierta en relación a culturaeducación e identidad, gratitud, formación integral y carácter sistémico e histórico (Añorga-Morales, 2014).

Aunque se justifica a la virtud, de inicio, como un origen genético, se considera que los aspectos educativos la fortalecen de forma notoria y sistemática (Vess et al., 2018). En la alfabetización tradicional, por ejemplo, se atribuye la virtud con la relación que el individuo tiene hacia la lectura y con el contexto del contenido del intelecto. La virtud en educación es formar ciudadanos de bien en su actuar, con afinidad al crecimiento humano (Balduzzi, 2015). La generación de conocimiento y la $\mathrm{CC}$ es una forma de diálogo a través de la trasmisión de información, de lo cual, deben surgir nuevos diálogos fructíferos en relación al conocimiento generado y publicado (Arriarán Cuéllar y Beuchot, 1999).

La virtud, la educación y la ciencia nunca resultan conceptos distantes (Barhr, 2017). Primero ofrecen una relación entre el individuo y la sociedad (Nadja Hermann, 2013). Las instituciones, a través de la educación y la ciencia intentan contribuir al desarrollo de la virtud, por tanto, ponen a disposición de sus investigadores científicos, legislaciones y estructuras formales acordes para que suceda, buscando con ello, la formación de valores interiorizados, deberes, responsabilidades prácticas, así como: virtudes institucionales, profesionales y ciudadanas (Escudero Muñoz, 2006).

La promoción de la potencialidad científica (PC) del individuo, sucede a través de la calificación de sus hábitos, así como registrando y desarrollando todos los aspectos que se identifican en el estado de sus capacidades y voluntades (Arriarán Cuéllar y Beuchot, 1999). El desarrollo científico, el aspecto institucional y la CC se vuelven un círculo complejo (García Delgado, Alonso y Jiménez, 2013), el cual se rige por su impacto y sus mediciones, que se convierten en exigencias para investigadores científicos por parte de las instituciones en donde se desempeñan o tienen relación científica, con lo cual se tiene muy en claro las condiciones de calidad científica (Maqueda Blasco et al., 2013).

La disciplina filosófica propone que la virtud debe ser observable, más no indica que deba ser medible. Sin embargo, al ser estudiado el tema desde una perspectiva científica hacia la generación de conocimiento, se vuelve necesaria la búsqueda de metodologías que permitan ofrecer resultados tangibles de su condición, con lo cual, se recurre a buscar la transferencia de aspectos meramente cualitativos hacia su registro cuantitativo.

La forma de construcción del parámetro de Virtud, consistió en el análisis y contrastación de diversos referentes, tanto clásicos como contemporáneos, encontrando una mayor consistencia y coherencia en la virtud aristotélica, la cual define tres elementos, mismos que son asociados con el perfil del investigador (Figura 1):

- Carácter (conocimiento y razón). Derivación de la enseñanza, de la pedagogía y/o epistemología, de la contemplación teórica y la 
racionalidad. Conocimiento del área o especialidad, así como, del quehacer, de los elementos, herramientas, técnicas, métodos y estrategias a utilizar en el estado del arte, investigación, análisis de hallazgos y la objetivación del conocimiento a través de publicaciones científicas.

- Hecho moral (acciones realizadas). Es la puesta en práctica de las habilidades investigativas y de comunicación científica que demuestra el investigador y que son aprendidas como un proceso de conocimiento y razón.

- Voluntad (intencionalidad). La inclinación que reviste al investigador para realizar su actividad conforme a sus intenciones. Le suman, la expectativa positiva en el desempeño científico, interés por compartir los resultados encontrados, así como, el reflejo de la sensibilidad ante las necesidades de los individuos y la satisfacción profesional y personal.

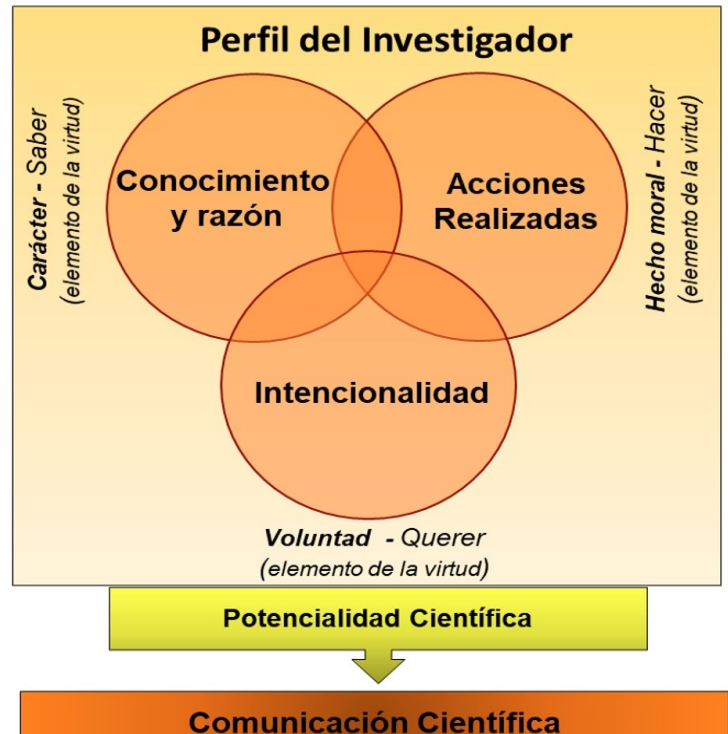

Figura 1. Elementos de la virtud visualizados en el perfil del investigador

\section{Metodología del estudio}

Los elementos metodológicos que sustentan esta investigación se resumen a continuación:

El enfoque del estudio es de tipo descriptivo-correlacional y de carácter no experimental, ya que no se manipularon variables y donde se utilizó un instrumento tipo encuesta para la recolección de los datos estadísticos cuantitativos.

El método utilizado fue el de análisis diferencial de los indicadores de virtud, PC y CC para los segmentos poblacionales, así como el estudio de la relación que los une.
La población de interés fue: investigadores (con grado de doctorado) de dos universidades públicas estatales, ubicadas en el estado de Chihuahua, México (Universidad Autónoma de Chihuahua y Universidad Autónoma de Ciudad Juárez), siendo las dos instituciones de mayor población en nivel superior, de las cuales se recolectaron datos a través de un instrumento tipo encuesta, aplicado de forma presencial y en formato electrónico (se incluye el instrumento en el apartado de Apéndices).

En ambas instituciones se identificaron 731 docentes con grado de doctorado que ejercen actividades de docencia e investigación, aplicándose un muestreo aleatorio que seleccionó a 183 participantes, siendo los parámetros del cálculo muestral los siguientes: (1) relación p/q de 80/20; (2) nivel de confianza de $95 \%$; (3) margen de error con un $7 \%$; y (4) población de 731 docentes.

- Hipótesis primaria: Los elementos fundamentales de la virtud (carácter, voluntad y hecho moral), manejados en la investigación como conocimiento, uso e interés, están asociados e influyen en la PC de la CC de los investigadores.

- Hipótesis secundarias (HS): los promedios en CC y en su PC no son iguales entre los investigadores según: su nivel de reconocimiento como investigador en el Sistema Nacional de Investigadores de México o SNI (HS1); el área científica de estudio como investigador del SNI (HS2); hayan sido o no investigadores del $\mathrm{SNI}$ en algún momento (HS3); hayan publicado en Scopus y/o Web of Science (HS4); y su tipo de contrato laboral (HS5).

\section{Análisis de resultados}

\subsection{Estadísticos descriptivos}

Los datos que conforman los elementos informativo-descriptivos sobre el perfil del profesorado participante en el estudio son los siguientes.

La edad promedio correspondió a 45 años, siendo 73 años la edad mayor y 29 la menor, siendo que el rango de edad con mayor frecuencia se encuentra entre los 35 y 45 años.

El tipo de contrato laboral se distribuyó en tres categorías: tiempo completo (90\%), por horaclase $(7.9 \%)$ y medio tiempo $(2.1 \%)$.

Dado que los investigadores participantes pueden o no tener reconocimiento como tales por parte del SNI (se reconocen cuatro niveles: Candidato, Nivel I, II y III), se observó que el $41.5 \%$ no lo tienen y $58.5 \%$ si son reconocidos por este sistema de evaluación (29.3\% en el Nivel I, 25.9 $\%$ en nivel de Candidato y $3.3 \%$ en nivel II). 
Las disciplinas científicas en las que participan los investigadores con reconocimiento del SNI, se dividieron en dos formas: (1) división disciplinar amplia: ciencias sociales y humanidades (25 $\%$ ), ciencias exactas (35\%) y sin reconocimiento en SNI (40\%); y (2) división disciplinar específica asignada por el propio SNI: ciencias sociales (15 $\%)$, Biotecnología y ciencias agropecuarias (11.3 $\%)$, Humanidades y ciencias de la conducta (10 $\%)$, Ingenierías (8.4\%), Medicina y ciencias de la salud $(7.9 \%)$, Biología y química $(5.4 \%)$ y, Físico Matemáticas y ciencias de la tierra (1.7\%).

\subsection{Análisis de resultados inferenciales}

Para realizar este tipo de análisis, se asumen los supuestos de linealidad y aditividad de los tres elementos de la virtud: conocimiento y razón, intencionalidad y acciones realizadas (esto es: conocimiento, interés y uso) y se considera que la PC viene dado por la suma lineal de los indicadores de los dominios disciplinares y los indicadores del carácter y perfil científico investigativo de los sujetos bajo estudio.

\subsubsection{Pertenencia al SNI con reconocimiento como investigador nacional}

Al comparar a investigadores con reconocimiento del SNI y los que no lo tienen, se observó que aquellos que no gozan de tal reconocimiento mostraron promedios más bajos, tanto en CC como en PC y, además, quienes si tienen el reconocimiento muestran resultados más altos, especialmente los de Nivel II $(C C=43.13$ y $P C=68.5)$. Los resultados más bajos se observaron en docentes que no son miembros del SNI $(\mathrm{CC}=34.48$ y $\mathrm{PC}=65.59)$.

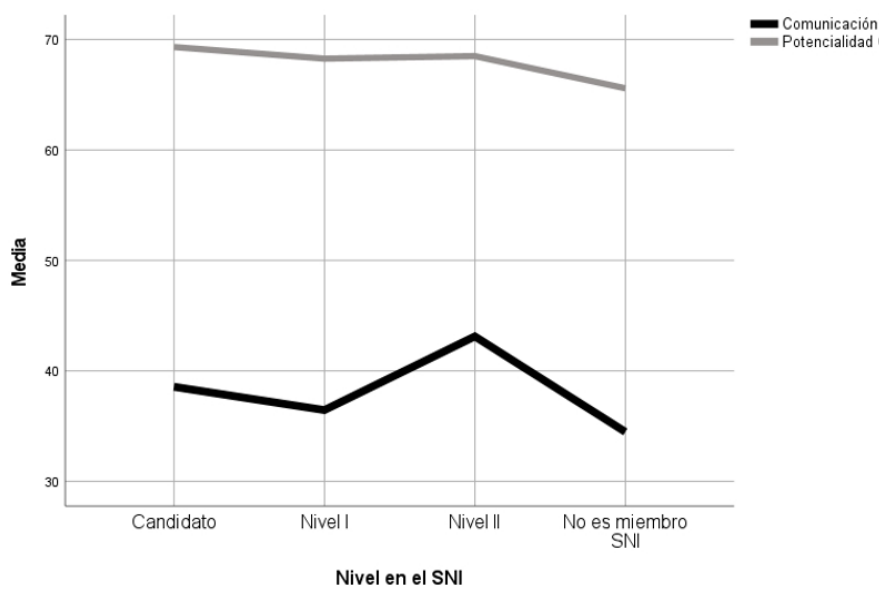

Figura 2. ANOVA entre $P C$ y $C C$ según su nivel en el SIN

Las diferencias entre el PC y la $\mathrm{CC}$, según el ANOVA realizado, resultaron estadísticamente significativas (CC sig=0.012 y $\mathrm{P}$ sig=0.022) y su apreciación gráfica se muestra en la Figura 2, por lo anterior se rechaza la hipótesis nula de igualdad de medias (PC y CC) entre los investigadores, según su tipo de pertenencia al SNI.

\subsection{2. Área disciplinar científica de pertenencia del investigador}

El análisis diferencial dentro del área disciplinar científica de pertenencia del investigador se realizó también con un ANOVA, pero además de considerar a los investigadores con reconocimiento del SNI se compararon dichas áreas con los datos de investigadores que no son miembros del SNI como si fueran un área independiente a las siete áreas disciplinares.

Con el ANOVA, se encontraron diferencias significativas entre ambas variables $C C($ sig $=0.00)$ y $P$ $($ sig $=0.018)$, siendo el área I. Físico Matemáticas y Ciencias de la Tierra la que arroja el promedio más alto en CC y estadísticamente diferente, usando la prueba de Tukey para el contraste de medias, con el área IV. Humanidades y Ciencias de la Conducta (junto con los investigadores no miembros del SNI), que mostraron los promedios más bajos.

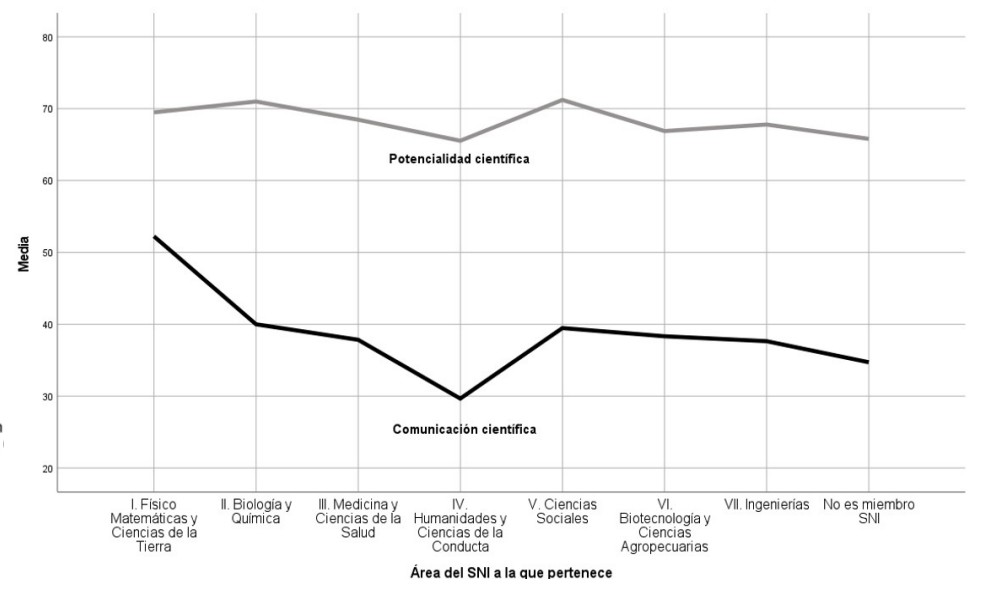

Figura 3. Prueba Tukey para el contraste de medias por área disciplinar del SIN

Para el caso del PC son las áreas V. Ciencias Sociales y II. Biología y Química, las que tienen los promedios más altos y de nuevo el área IV. Humanidades y Ciencias de la Conducta, al igual que los profesores no miembros del SNI, muestran los promedios más bajos, sin embargo, aplicando la prueba de Tukey para el contraste de medias, sólo se detectaron diferencias estadísticas significativas entre el área V. Ciencias Sociales y los profesores que no son miembros del SNI, según se corrobora en la Figura 3. Por lo anterior, se rechaza la hipótesis nula de igualdad de 
medias (CC y PC) entre los profesores investigadores, según su área de pertenencia dentro del SNI.

\subsubsection{Pertenencia actual o anterior al SNI}

Este indicador identifica aquellos investigadores que en otro tiempo tuvieron distinción como investigadores nacionales del SNI y que por diversas circunstancias no han logrado su reingreso 0 permanencia en el mismo. Los resultados obtenidos son: no ha tenido la distinción en ningún momento $31.4 \%$ ), perdió la permanencia y se conserva sin reingreso $(13.8 \%)$ y actualmente tiene la distinción (54.8\%).

En este apartado se considera la hipótesis secundaria de que la pertenencia al SNI en algún momento del tiempo, es un factor que influye en la PC y la CC, arrojando promedios diferentes en los tres casos considerados: (1) es miembro actual; (2) ha sido miembro en el pasado; y (3) no ha sido miembro. De acuerdo a los resultados obtenidos, se observa que los profesores con pertenencia actual tienen los promedios más altos, seguidos por los que en el pasado han estado en el SNI y en el nivel más bajo profesores que no han pertenecido al SNI.

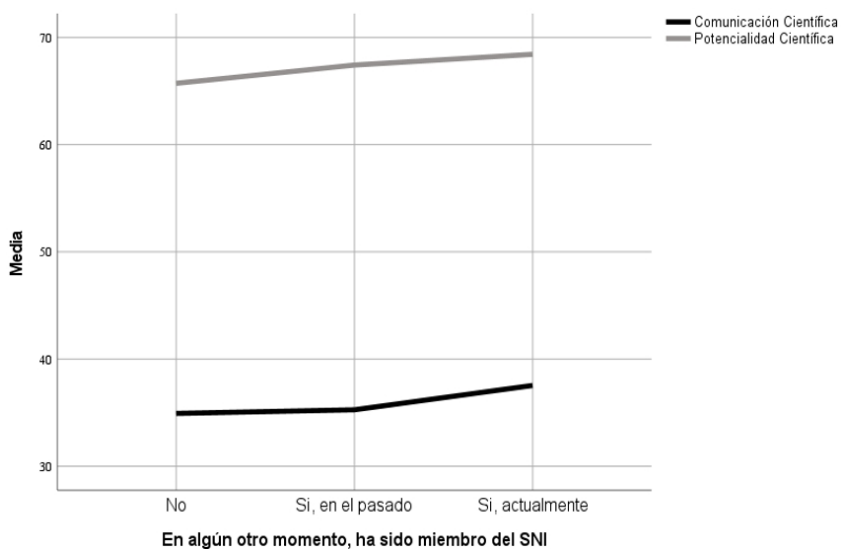

Figura 4. ANOVA entre PC y CC según nivel SNI a través del tiempo

Sin embargo, al ejecutar el ANOVA de un factor, no se encontraron diferencias estadísticas significativas entre estos tres grupos de profesores, tanto para la CC (sig=0.137), como para la PC $($ sig $=0.066)$, según se aprecia gráficamente en la Figura 4.

\subsubsection{Publicaciones en revistas científicas arbitradas e indizadas incluidas en Scopus y/o Web of Science}

Independientemente de que los investigadores pertenezcan o no al SNI, en este caso se planteó la hipótesis de que los investigadores que publican artículos científicos en revistas arbitradas e indizadas incluidas en Scopus y/o Web of Science, tienen diferentes promedios tanto en CC como en PC.

Con los resultados de la prueba "t" para muestras independientes se comprueba la hipótesis de trabajo, al reportarse diferencias estadísticas altamente significativas tanto en CC (sig=0.000) como en PC (sig $=0.000$ ), lo cual también puede apreciarse en el diagrama de cajas de la Figura 5.

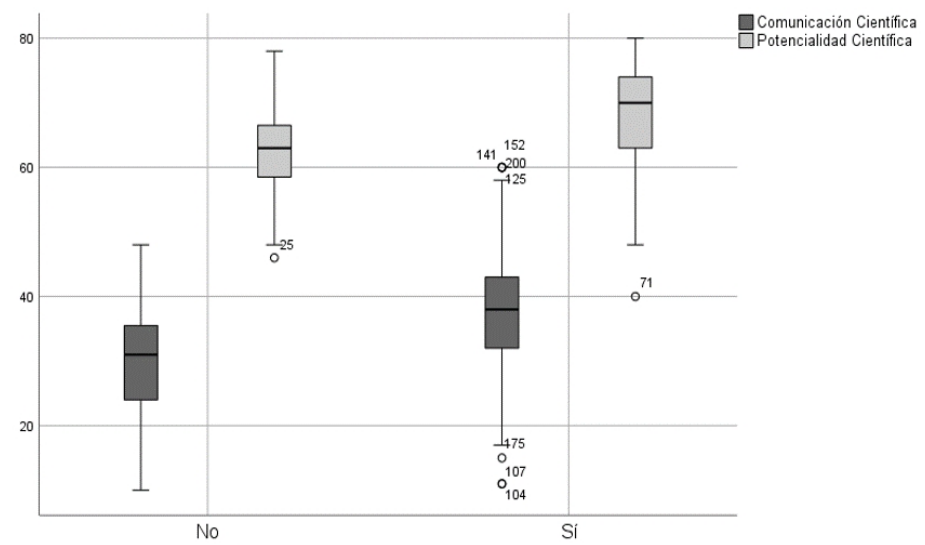

Figura 5. Realización de publicaciones en revistas arbitradas e indizadas (Scopus y Web of Science)

\subsubsection{Tipo de contrato laboral de los investigadores}

La comprobación de la última hipótesis secundaria que se asumió en este trabajo, donde se suponía que los investigadores de tiempo completo arrojarían mejores promedios, tanto en CC como en PC, se aplicó el ANOVA de un factor junto con la prueba de Tukey para la contratación de medias, encontrándose resultados descriptivos que contradicen la hipótesis secundaria, al ser los investigadores de hora clase los que arrojan los promedios más altos tanto en CC como en PC, seguidos por los investigadores de tiempo completo y con los promedios más bajos, los investigadores de medio tiempo.

Según los resultados del ANOVA, se rechaza la hipótesis nula de igualdad de medias en CC $(\mathrm{sig}=0.050)$ y también en PC (sig=0.012), con un $95 \%$ de confianza, por lo que procede la prueba de Tukey de separación de medias.

En la prueba de Tukey de separación de medias, sólo se detectaron diferencias estadísticas significativas en CC entre los profesores hora clase (más altos promedios) con los medios tiempos (más bajos promedios), no así con los tiempos completos. Para el caso de la PC, si se detectaron diferencias significativas entre los tres tipos 
de profesores, siendo los profesores horas clase los promedios más altos, seguidos por los tiempos completos y al final los medios tiempos con los promedios más bajos (Figura 6).

De acuerdo al resumen de la contrastación de las hipótesis del trabajo (Tabla I), es posible observar la condición de la hipótesis principal y de las hipótesis secundarias, de las cuales se derivan las siguientes conclusiones: 1) los elementos de la virtud que demuestra el investigador, son determinantes en su nivel de PC y capacidad de CC; (2) A mayor nivel del investigador como miembro del SNI, mayor es su demostración de la virtud proyectada en su PC y CC; (3) La demostración de la virtud de los investigadores en su PC y CC varia de forma significativa entre las ciencias exactas y las ciencias sociales y humanidades, demostrando mayor nivel los primeros; (4) Tener el reconocimiento como investigador por parte de SNI, representa mejores niveles virtud de PC y CC, incluso, el haber pertenecido se posiciona mejor que en comparación con quienes no lo han tenido; (5) la publicación en revistas de impacto arbitradas e indizadas, representa un elemento de reconocimiento de la virtud a través de mayores indicadores en PC y CC; y (6) los mejores tipos de contrato laboral (tiempo completo) no es determinante para demostrar mejores niveles de virtud.

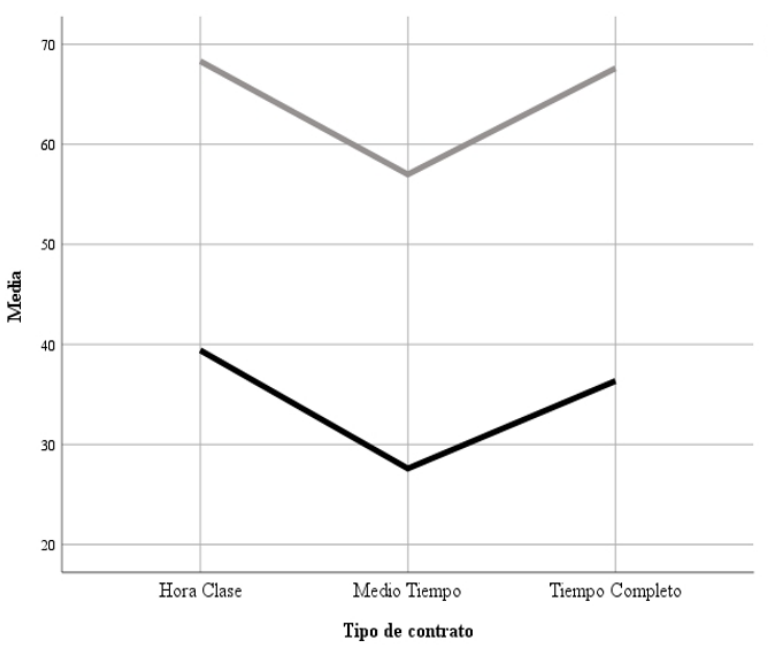

Figura 6. Prueba Tukey de separación de medidas según tipo de contrato

\begin{tabular}{|c|c|c|c|c|c|}
\hline$N^{\circ}$ & Hipótesis nula $(\mathrm{Ho})$ & Hipótesis alterna(Ha) & Análisis o Prueba & Sig. & Decisión \\
\hline $\mathrm{Pp}$ & $\begin{array}{l}\text { Los elementos de la virtud } \\
\text { (conocimiento, uso e interés) no } \\
\text { están asociados y no influyen en } \\
\text { la PC científica de los } \\
\text { investigadores }\end{array}$ & $\begin{array}{l}\text { Los elementos de la virtud } \\
\text { (conocimiento, uso e interés) } \\
\text { están asociados e influyen en la } \\
\text { PC científica de los } \\
\text { investigadores }\end{array}$ & $\begin{array}{l}\text { Análisis de correlación } \\
\text { y regresión }\end{array}$ & 0.00 & $\begin{array}{l}\text { Retener la } \\
\text { hipótesis de } \\
\text { trabajo o } \\
\text { hipótesis } \\
\text { alterna }\end{array}$ \\
\hline 1 & $\begin{array}{l}\text { Los promedios en CC y en PC } \\
\text { son iguales entre los profesores } \\
\text { según su membresía SNI }\end{array}$ & $\begin{array}{l}\text { Los promedios en CC y en PC } \\
\text { no son iguales entre los } \\
\text { investigadores según su } \\
\text { membresía SNI }\end{array}$ & $\begin{array}{l}\text { ANOVA de un Factor y } \\
\text { Prueba de Tukey para } \\
\text { separación de medias }\end{array}$ & $\begin{array}{l}C C \\
0.012 \\
P \\
0.022\end{array}$ & $\begin{array}{l}\text { Retener la } \\
\text { hipótesis de } \\
\text { trabajo o } \\
\text { hipótesis } \\
\text { alterna }\end{array}$ \\
\hline 2 & $\begin{array}{l}\text { Los promedios en CC y en PC } \\
\text { son iguales entre los profesores } \\
\text { según el área de pertenencia del } \\
\text { SNI }\end{array}$ & $\begin{array}{l}\text { Los promedios en CC y en PC } \\
\text { no son iguales entre los } \\
\text { profesores según el área de } \\
\text { pertenencia del SNI }\end{array}$ & $\begin{array}{l}\text { ANOVA de un Factor y } \\
\text { Prueba de Tukey para } \\
\text { separación de medias }\end{array}$ & $\begin{array}{l}C C \\
0.000 \\
P \\
0.018\end{array}$ & $\begin{array}{l}\text { Retener la } \\
\text { hipótesis de } \\
\text { trabajo o } \\
\text { hipótesis } \\
\text { alterna }\end{array}$ \\
\hline 3 & $\begin{array}{l}\text { Los promedios en CC y en PC } \\
\text { son iguales entre los profesores } \\
\text { según hayan pertenecido al SNI } \\
\text { en algún momento }\end{array}$ & $\begin{array}{l}\text { Los promedios en CC y en PC } \\
\text { no son iguales entre los } \\
\text { profesores según hayan } \\
\text { pertenecido al SNI en algún } \\
\text { momento }\end{array}$ & $\begin{array}{l}\text { ANOVA de un Factor y } \\
\text { Prueba de Tukey para } \\
\text { separación de medias }\end{array}$ & $\begin{array}{l}\mathrm{CC} \\
0.137 \\
P \\
0.066\end{array}$ & $\begin{array}{l}\text { Retener la } \\
\text { hipótesis nula }\end{array}$ \\
\hline 4 & $\begin{array}{l}\text { Los promedios en CC y en PC } \\
\text { son iguales entre los profesores } \\
\text { según hayan publicado en } \\
\text { Scopus y/o Web of Science }\end{array}$ & $\begin{array}{l}\text { Los promedios en CC y en PC } \\
\text { no son iguales entre los } \\
\text { profesores según hayan } \\
\text { publicado en Scopus y/o Web } \\
\text { of Science }\end{array}$ & $\begin{array}{l}\text { Prueba "t" para } \\
\text { muestras } \\
\text { independientes }\end{array}$ & $\begin{array}{l}C C \\
0.000 \\
P \\
0.000\end{array}$ & $\begin{array}{l}\text { Retener la } \\
\text { hipótesis de } \\
\text { trabajo o } \\
\text { hipótesis } \\
\text { alterna }\end{array}$ \\
\hline 5 & $\begin{array}{l}\text { Los promedios en CC y en PC } \\
\text { son iguales entre los profesores } \\
\text { según su tipo de contrato laboral }\end{array}$ & $\begin{array}{l}\text { Los promedios en CC y en PC } \\
\text { no son iguales entre los } \\
\text { profesores según su tipo de } \\
\text { contrato laboral }\end{array}$ & $\begin{array}{l}\text { ANOVA de un Factor y } \\
\text { Prueba de Tukey para } \\
\text { separación de medias }\end{array}$ & $\begin{array}{l}C C \\
0.050 \\
P \\
0.012\end{array}$ & $\begin{array}{l}\text { Retener la } \\
\text { hipótesis de } \\
\text { trabajo o } \\
\text { hipótesis } \\
\text { alterna }\end{array}$ \\
\hline
\end{tabular}

Tabla I. Resumen de contrastación de hipótesis principal e hipótesis secundarias 


\section{Discusión y conclusión}

El proceso de identificación y desarrollo de investigadores en universidades mexicanas afronta condiciones críticas, en particular sobre la forma como se incorporan y se desarrollan sus actividades de investigación, a través del reclutamiento y movilidad en las comunidades científicas (Rivas Tovar, 2004). Los procesos de selección de investigadores no consideran a la virtud como un elemento nato o natural (Vess, 2018); por la otra parte, el desarrollo de la virtud académica e investigativa durante su proceso laboral, se ha caracterizado por no suceder de manera formal y sistemática, sino por un proceso de aprendizaje no planeado, basado en la voluntad individual (Balduzzi, 2015).

Los resultados de investigación comprueban las diferencias en virtud entre los docentes que han obtenido reconocimiento formal como investigadores (la cual varia positivamente en lo que crece su nivel), en comparación de los que no lo tienen. Esto tiene como implicaciones futuras, que las instituciones educativas y de investigación mexicanas deberán integrar programas formales de desarrollo de la virtud en la conformación de autoridades en el mundo científico, especialmente a través de la definición de políticas públicas sobre vocaciones científicas: (1) selección del ingreso basado en los elementos de la virtud a través de la creación de paradigmas profesionales (Acuña Gamboa et al., 2017; Barhr, 2017); (2) generación de mecanismos de promoción de la virtud para docentes que guarde interés en obtener el reconocimiento como investigadores desde etapas laborales tempranas (Castillo Sánchez, 2014; Berg, 2020); y (3) fomento en el fortalecimiento de los elementos de la virtud en investigadores con reconocimiento formal como tales, buscando incrementar la permanencia y crecimiento en su nivel (Gutiérrez Serrano, 2014).

\section{Referencias}

Acuña Gamboa, Luis Acuña; Barraza Macías, Arturo; Jaik Dipp, Adla (2017). Formación de investigadores educativos en Latinoamérica: hacia la construcción de un estado del arte. México: REDIE, 2017.

Añorga-Morales, Julia Aurora (2014). La educación avanzada y el mejoramiento profesional y humano. // Varona. ISSN 0864-196X. 48 (enero-junio 2014) 19-31.

Armas Faris, Fernando (2011). Virtudes epistémicas y conocimiento científico en la obra de Mariano Artigas. Pamplona: Universidad de Navarra, Facultad Eclesiástica de Filosofía, 2011. Tesis doctoral.

Arriarán Cuéllar; Samuel y Beuchot, Mauricio (1999). Virtudes, valores y educación moral: Contra el paradigma neoliberal. México: Universidad Pedagógica Nacional, 1999.

Balduzzi, Emanuele (2015). El cultivo de las virtudes a través de los episodios de aprendizaje situados (EAS). Teoría de la Educación. // Revista Interuniversitaria. ISSN 23865660. 27:1, 155-167. http://doi.org/10.14201/teri.13079

Barhr, Jason (2017). The Varieties of Character and Some Implications for Character Education. // Journal of Youth and Adolescence. ISSN 1573-6601. 46:6 (March, 2017) 1153-1161. https://doi.org/10.1007/s10964-017-0654-z

Barrantes Montero, Luis Guillermo (2013). Ethical responsibility of teachers and students from a humanistic perspective. // Revista Electrónica Educare. ISSN 1409-4258. 17:1 (enero-abril 2011) 29-45.

Berg, Henrik (2020). Virtue Ethics and Integration in Evidence-Based Practice in Pshychology. // Frontiers in Psychology. ISSN 1664-1078. 11:258. (February 2020) 15. https://doi.org/10.3389/fpsyg.2020.00258

Burlando, Giannina (2012). Historia de la virtud que buscamos: del aristotelismo a la modernidad. // Acta Scientiarum: Education Maringá. ISSN 2158-5201. 34:1 (jan.-june 2012) 1-9. http://doi.or/10.4025/actascieduc .v34i1.15351

Castillo Sánchez, Mauricio (2014). Identificación de estrategias para la formación de investigadores desde la escuela. // Studiositas. ISSN 0188-7653. 12:25 enero-junio 2004) 89-113.

Escudero Muñoz, Juan M. (2006). Compartir propósitos y responsabilidades para una mejora democrática de la educación. // Revista de Educación. ISSN 1988-592X. 339: 2 (2006) 19-42.

Fowers, Blaine J.; Carroll, Jason S.; Leonhardt, Nathan D.; Cokelet, Bradford (2020). The Emerging Science of Virtue. // Perspectives on Psychological Science. ISSN 1745-6916. 16:1 (2020) 118-147. https://doi.org/10.1177/ 1745691620924473

Garcés Giraldo, Luis Fernando; Giraldo Zuluaga, Conrado (2013). La virtud: la recta razón en el profesional que experimenta con animales. // Civilizar: Ciencias Sociales y Humanas. ISSN 1657-8953.13:24 (2013) 181-191. https://doi.org/10.22518/16578953.122

García Delgado, José Luis; Alonso, José Antonio; Jiménez, Juan Carlos, Coord. (2013). El español, lengua de comunicación científica. Barcelona: Ariel; Fundación Telefónica, 2013.

Gutiérrez Serrano, Norma Georgina (2014). Producción de conocimiento y formación de investigadores // Sinéctica. ISSN 2007-7033. 43 (julio-diciembre 2014) 1-16.

Maqueda Blasco, Jerónimo; Gamo González, M ${ }^{a}$ Fe; Cortés Barragán, Rosana; Veiga-Cabo, Jorge (2013). Estructura y contenidos de la comunicación y redacción de artículos científicos. Med. Segur. Trab. ISSN 1989-7790. 59:230 (enero-marzo 2013) 159-170.

Nadja Hermann, Mara (2013). Virtud y amor en Rousseau. Porto Alegre, Brasil: Associação Sul-Rio-Grandense de Pesquisadores em História de Educação (ASPHE), 2013.

Näsman, Yvonne; Nyholm, Linda (2020). The Movement of Virtue from Ethos to Action. // Nursing Philosophy. ISSN 1466-769X. 22:2 (December 2020) e12339. https://doi. org/10.1111/nup.12339

Rivas Tovar, Luis Arturo (2004). La formación de investigadores en México. // Perfiles Latinoamericanos. ISSN: 01887653. 12:25 (enero-junio 2004) 89-113

Tomić, Vicko; Juljan, Ivan; Marušić, Ana (2021). Perspectives of key stakeholders on essential virtues for good scientific prac-tice in research areas. // Accountability in Research (prepint) (2020). ISSN 0898-9621. https://doi.org/10. 1080/08989621.2021.190073

Vélez-Ramírez, Amparo (2008). La adquisición de hábitos como finalidad de la educación superior. // Teoría de la Educación. ISSN 0123-1294. 11:1 (2008) 167-180.

Vess, Mattew; Brokker, Rebecca J.; Stichter, Matt (2018). Genes and Virtue: Exploring how Heritability Beliefs Shape 
Conceptions of Virtue and its Development. // Behavior Genetics. ISSN 1573-3297. 49:2 (June 2018) 168-174. https://doi.org/10.1007/s10519-018-9908-0.
Enviado: 2021-02-18. Segunda versión: 2021-06-15 Aceptado: 2021-09-10.

\section{Apéndice I: Instrumento de recolección de datos}

El presente cuestionario de carácter anónimo, forma parte de la investigación que corresponde a la tesis "Comunicación científica potencializada a través de elementos de la virtud" del Doctorado en Educación Artes y Humanidades de la Universidad Autónoma de Chihuahua. (Perteneciente al PNPC-CONACYT). El instrumento permitirá conocer si ciertos elementos presentes en los dominios del docente, potencializan la comunicación cientíica.

Las respuestas serán empleadas sólo con fines estadísticos, por lo cual le solicitamos conteste de una manera abierta y con la mayor veracidad posible.

I. Datos generales del encuestado

1. Edad:

2. Institución en que labora:

3. Facultad o Departamento en que labora:

4. ¿De qué tipo es su contrato como docente? [ Hora / Clase Medio / Tiempo / Tiempo / Completo ]

5. Si es miembro del SNI, ¿cuál es su nivel? [ Candidato / I II / III ]

6. Si es miembro del SNI, ¿a qué área pertenece? [ I. Físico Matemáticas y Ciencias de la Tierra / II. Biología y Química / III. Medicina y Ciencias de la Salud / IV. Humanidades y Ciencias de la Conducta / V. Ciencias Sociales / VI. Biotecnología y Ciencias Agropecuarias VII. Ingenierías ]

7. Si no es miembro del SNI, ¿en algún otro momento lo ha sido? [ Sí / No ]

8. ¿Cuenta con perfil PRODEP? [ Sí / No ]

Comunicación de la ciencia

Instrucciones: Marque la opción que corresponda a su situación actual, de la misma manera de respuesta a las preguntas abiertas.

9 ¿Cómo define la comunicación científica?

10 ¿Realiza publicaciones en revistas arbitradas e indizadas (pertenecientes a Scopus y Web of Science)? [ Sí / No ]

11 ¿Cuáles son las razones de su respuesta anterior?

12 ¿Cuáles razones considera que limitan la comunicación científica en su institución?

II. Dominios disciplinares

Instrucciones: Marque la opción de dominios en su área o disciplina que mayormente corresponda a su situación actual.

\begin{tabular}{lc}
\hline Grado de valoración o nivel de acuerdo & Valor \\
\hline Totalmente en desacuerdo & (0) \\
\hline Bastante en desacuerdo & (1) \\
\hline En desacuerdo & (2) \\
\hline De acuerdo & (3) \\
\hline Bastante de acuerdo & (4) \\
\hline Totalmente de acuerdo & (5) \\
\hline
\end{tabular}

\begin{tabular}{|c|c|c|c|c|c|c|}
\hline \multirow{2}{*}{$\begin{array}{l}\text { Nivel de Valoración } \\
\text { 13. Conozco las problemáticas y retos actuales que atañen a la disciplina que estu- } \\
\text { dio. }\end{array}$} & \multicolumn{3}{|c|}{ Totalmente en desacuerdo } & \multicolumn{3}{|c|}{ Totalmente de acuerdo } \\
\hline & (0) & (1) & (2) & (3) & (4) & (5) \\
\hline 14. Estoy enterado de las tendencias de la disciplina que estudio. & (0) & (1) & (2) & (3) & (4) & (5) \\
\hline 15. Busco una actualización profesional autodidacta. & (0) & (1) & (2) & (3) & (4) & (5) \\
\hline $\begin{array}{l}\text { 16. Prefiero una actualización profesional a través de eventos institucionales y pro- } \\
\text { gramas estructurados. }\end{array}$ & (0) & (1) & (2) & (3) & (4) & (5) \\
\hline 17. Adopto el proceso de aprendizaje como una constante de toda la vida. & (0) & (1) & (2) & (3) & (4) & (5) \\
\hline $\begin{array}{l}\text { 18. Soy miembro, estoy suscrito o utilizo algún sitio web referente a la disciplina que } \\
\text { estudio. }\end{array}$ & (0) & (1) & (2) & (3) & (4) & (5) \\
\hline 19. Cuento con alguna red de contactos de colegas profesionales. & (0) & (1) & (2) & (3) & (4) & (5) \\
\hline 20. Tengo acceso o cuento con la tecnología idónea para investigar y/o actuali- & (0) & (1) & (2) & (3) & (4) & (5) \\
\hline
\end{tabular}
zarme.

III. Carácter y perfil cientifico-investigativo

Instrucciones: Marque la opción que mejor define el impulso de su carácter y perfil en investigación y ciencia.

\begin{tabular}{lc}
\hline Grado de valoración o nivel de acuerdo & Valor \\
\hline No, nunca. muy bajo & (0) \\
\hline Muy poco, casi nunca & (1) \\
\hline Poco, alguna vez & (2) \\
\hline
\end{tabular}




\begin{tabular}{ll}
\hline Mucho, frecuentemente & (3) \\
\hline Bastante, muy frecuente & (4) \\
\hline Sí, siempre. muy alto & (5) \\
\hline
\end{tabular}

\begin{tabular}{|c|c|c|c|c|c|c|}
\hline \multirow{2}{*}{$\begin{array}{l}\text { Nivel de valoración } \\
\text { 21. Tiendo a apasionarme por mi desempeño científico. }\end{array}$} & \multicolumn{3}{|c|}{ Totalmente en desacuerdo } & \multicolumn{3}{|c|}{ Totalmente de acuerdo } \\
\hline & (0) & (1) & (2) & (3) & (4) & (5) \\
\hline 22. Siento el deber de comunicar los resultados encontrados en mis investigaciones. & (0) & (1) & (2) & (3) & (4) & (5) \\
\hline 23. Soy sensible ante las necesidades locales e internacionales de la población. & (0) & (1) & (2) & (3) & (4) & (5) \\
\hline 24. Suelo indagar, consultar y manejarme éticamente en la actividad científica. & (0) & (1) & (2) & (3) & (4) & (5) \\
\hline 25. Me atrae mi superación en la actividad científica y la satisfacción personal. & (a) & (1) & (2) & (3) & (4) & (5) \\
\hline $\begin{array}{l}\text { 26. Me acerco a nuevas tecnologías, colegas e instituciones editoriales, de ciencia y tec- } \\
\text { nología que facilitan o me pueden auxiliar en la comunicación científica. }\end{array}$ & (0) & (1) & (2) & (3) & (4) & (5) \\
\hline 27. Tengo interés por el estatus y el reconocimiento institucional y de comunidad científica. & (1) & (1) & (2) & (3) & (4) & (5) \\
\hline $\begin{array}{l}\text { 28. Me atraen los beneficios que involucran el acceso al manejo de recursos y materiales, } \\
\text { así como la percepción de mejor futuro laboral, adquisitivo, personal y profesional. }\end{array}$ & (a) & (1) & (2) & (3) & (4) & (5) \\
\hline
\end{tabular}

Acercamiento a la investigación y ciencia

Instrucciones: Señale la alternativa que mejor define su acercamiento a la ciencia e investigación.

\begin{tabular}{lc}
\hline Grado de valoración o nivel de acuerdo & Valor \\
\hline Nada, poco & (1) \\
\hline Regular & (2) \\
\hline Suficiente, mucho & (3) \\
\hline
\end{tabular}

\section{Acercamiento a la investigación y la ciencia}

\begin{tabular}{|c|c|c|c|c|c|c|c|c|}
\hline \multicolumn{3}{|c|}{ Conozco } & \multicolumn{3}{|c|}{ He utilizado } & \multicolumn{3}{|c|}{ Tengo interés } \\
\hline (1) & (2) & (3) & (1) & (2) & (3) & (1) & (2) & (3) \\
\hline (1) & (2) & (3) & (1) & (2) & (3) & (1) & (2) & (3) \\
\hline (1) & (2) & (3) & (1) & (2) & (3) & (1) & (2) & (3) \\
\hline
\end{tabular}

29. Aproximación con la estructura de un proyecto de investigación: título, antecedentes, problema de investigación, variables, hipótesis o preguntas científicas, objetivos, métodos, actividades, referencias.

30. Acercamiento en la preparación de un artículo científico para publicación (planear, recolectar y organizar información; organizar pensamientos mediante outlines, mapas conceptuales y el desarrollo de tablas y figuras; escritura del primer borrador; revisión de organización y lógica, de fraseo y estilo, así como de gramática y puntuación).

31. Aplicación sistemática en el uso del método IMRyD e incluso con apoyo de organismos como ISO, ISI, Scopus, CEN, AENOR, AFNOR, COPANT etc., en cuanto a las normas para el contenido y la estructura de artículos científicos, resúmenes y referencias bibliográficas.

32. Relación con gestores bibliográficos que me permite almacenar y gestionar referencias bibliográficas, como con Mendeley, Endnote, tgt, RefWorks, Citeulike, etc.; con gestores como OJS y su funcionalidad de las alertas por correo electrónico y/o páginas web de terminología sobre gestión de la información.

33. Cercanía con software que permiten procesar datos, crear matrices y visualizar información a través de redes y grafos, como Bibexcel, Ucinet, Pajek, Netdraw, etc.; el lenguaje HTML para establecer hiperenlaces; el desarrollo un Crowler (araña web) para recolectar una muestra de artículos, etc.

34. Aproximación con la demarcación entre la publicación en acceso abierto y los sistemas de revisión previa como las propuestas de self-archiving que puede contener las versiones preprint, las copias idénticas de las postprint, o artículos e-prints aunque en un formato diferente, en repositorios como ArXiv.

35. Relación con el uso, divulgación o comunicación de artículos que además del texto, ofrecen gráficos, simulaciones computacionales, bases de datos, etc. que pueden integrarse con el resto de las prácticas de e-research.

36. Acercamiento con indicadores métricos de la información, tales como: factor de impacto, $\mathrm{H}-$ Index, el índice de inmediatez, SNIP, cuartiles, índice G; índices en fuentes de datos como ISI Web of Science, Scopus y Google Metrics; informes de Science Citation Index, Ranquin de Shangai, QS, Webometrics Google Scholar Metrics; plataformas y redes sociales académicas como Mendeley, Research Gate, ResearchID; bases de datos y agregadores como: DOAJ, EBSCO, Latindex, RedAlyC, IRESIE, etc.

37. Relación que se tiene con las Almétricas o indicadores alternativos basados en la Web 2.0., encontrados en plataformas como Citeulike, Connotea, o citas en blogs; con herramientas como altmetric.com, Plum Analytics, Science Card, Citedin o Impact Story.

38. Acercamiento con prácticas como la coautoría inflada o las publicaciones fragmentadas, las publicaciones predatorias y la diferenciación entre las publicaciones de calidad de las que no lo son.

Comentarios adicionales: y elementos de virtud en investigadores mexicanos. // Ibersid. 15:2 (jul.-dic. 2021) 13-21. 
